\section{Cahiers de Narratologie}

Analyse et théorie narratives

$10.1 \mid 2001$

La voix narrative

\title{
Una meditaciòn ou le récit d'un « arrangeur »
}

\section{Denise Boyer}

\section{OpenEdition}

\section{Journals}

Electronic version

URL: http://journals.openedition.org/narratologie/6946

DOI: 10.4000/narratologie.6946

ISSN: 1765-307X

\section{Publisher}

LIRCES

\section{Printed version}

Date of publication: 1 January 2001

Number of pages: $243-251$

ISBN: 2914561032

ISSN: 0993-8516

\section{Electronic reference}

Denise Boyer, "Una meditaciòn ou le récit d'un « arrangeur »", Cahiers de Narratologie [Online], 10.1 | 2001, Online since 24 October 2014, connection on 24 February 2021. URL: http://journals.openedition.org/ narratologie/6946 ; DOI: https://doi.org/10.4000/narratologie.6946 


\title{
UNA MEDITACIÓN OU LE RÉCIT D'UN « ARRANGEUR »
}

\author{
Denise BOYER \\ Université d'Orléans
}

Una meditación ${ }^{1}$ est le seul roman de Juan Benet doté d'un narrateur intradiégétique unique: nulle autre voix n'intervient donc pour « compléter » ou « corriger » son récit. Qui plus est Juan Benet a pris soin d'expliquer que ce narrateur «ne dit pas la vérité », qu'il «se contredit», et qu'il est "l'auteur ou l'instigateur de plusieurs des drames $»^{2}$ qu'il raconte. La tâche du lecteur s'apparente donc à une gageure : comment s'orienter en effet dans un récit mensonger, et de surcroît extrêmement touffu, lacunaire, à la chronologie chaotique, constamment interrompu par de pesantes digressions, et totalement dépourvu d'alinéas qui marqueraient au moins des articulations dans le discours? Et il faut encore ajouter que l'auteur n'ayant pas coutume de manifester une telle sollicitude à son lecteur, on peut se demander si ses indications sont tout à fait fiables. C'est pourquoi on a tenté ici, avant de se livrer à la lecture " policière " suggérée par Benet, de dégager du seul texte une interprétation globale, sinon de la "vérité » fictionnelle, du moins de la personnalité du narrateur.

${ }^{1}$ Barcelone, 1970, Seix Barral, «Biblioteca breve », 329 p. Toutes les citations renvoient à cette édition.

${ }^{2}$ Es el discurso de un señor, que es un joven antes de la guerra [...] Este señor se equivoca, confunde, y, sobre todo, como todo narrador de muchas cosas, no dice la verdad y produce en su propio discurso sus insidias y, por lo tanto, se contradice. No creo que haya acertado en retratar indirectamente, porque a confesión propia no lo ha de decir, que es un bellaco y autor o instigador de algunos de los dramas de que consta el argumento. "Encuentro con Juan Benet", Antonio Nuñez, Ínsula, n²69, avril 1969, p. 4. 
La fiction de Una meditación est constituée des souvenirs d'un narrateur anonyme originaire de Región. Amoureux dès l'enfance de sa cousine Mary, il se lie par son intermédiaire aux deux fils de la famille Ruan, Enrique et Jorge, et à leur professeur Julián avec qui Mary se fiance bientôt. Juste avant leur mariage, elle fait une fugue avec un jeune homme fortuné qu'elle a connu chez les Ruan, Carlos Bonaval. La guerre civile a ensuite pour conséquences la disparition d'Enrique Ruan, qui combattait dans le camp républicain, et l'exil de Julián et Mary. Quant au narrateur qui étant donné son jeune âge n'avait pu ni séduire Mary ni prendre part à la guerre, il est si profondément affecté par la perte de son « mentor » et « seul ami » Enrique (p. 69) et de sa bien-aimée que cela détruit à tout jamais ses possibilités de maturation et de bonheur personnel. Quand il revient ensuite à Región pour de brefs séjours occasionnels, il n'y rencontre qu'hostilité : en particulier, dans les années 60 , lors d'un hommage posthume au poète Jorge Ruan, victime d'un assassinat non élucidé. Il y retrouve Bonaval, Mary qui s'est remariée en Amérique, et Cayetano Corral qui a été son ami avant de s'éloigner de lui pour se lier avec Jorge Ruan puis avec Carlos Bonaval. Et c'est à cette occasion qu'il entend Mary, en conversation avec les deux hommes, prononcer "l'épithète ": un terme péjoratif faisant allusion à la "passivité ", qu'il prend pour une critique à son égard 3 . Après la mort de Mary qui survient peu de temps après, sa maison est incendiée et là encore les coupables ne sont jamais découverts. Et le récit s'achève sur l'évocation d'une nouvelle aventure amoureuse de Carlos Bonaval, dont la maîtresse sombre dans la folie.

On remarque évidemment que l'essentiel de la fiction fait apparaître le narrateur comme une victime : de Mary qui l'a

${ }^{3}$ Ay, fue un epíteto nada más, un epíteto que salió de sus labios, cruelmente preciso y solitariamente pronunciado, $[\ldots]$ pero $[\ldots]$ cuanto más pensaba en él, imaginando y reconstruyendo todas las conversaciones posibles que sin ninguna referencia hacia mi provocaran por azar la expresión de la palabra a mi paso, más evidente me parecía que había sido pronunciada con toda intención, engendrado en el ánimo de una mujer y, de entre todas las mujeres de mi entorno, precisamente en Mary. (p. 235-329). 
dédaigné à la fois comme mari et comme amant, et même comme ami au profit de Jorge Ruan; de Cayetano Corral qui lui a repris son amitié ; et de Jorge Ruan puis de Carlos Bonaval, qui lui ont en quelque sorte dérobé cette même amitié. Cette thématique nettement "persécutoire» (le lexique aussi est symptomatique ${ }^{4}$ ) laisse évidemment suspecter une petite paranoïa (étant entendu que « les paranoïaques aussi ont leurs ennemis $»^{5}$ ); or cette hypothèse de lecture «psychiatrique » est étayée par les caractéristiques les plus frappantes du récit. Il y a d'abord la nature et la teneur de ses deux principales modalités : récit d'événements et digressions. On observe en effet, dans le récit d'événements, une disproportion entre la brièveté des passages concernant directement le narrateur et la longueur de ceux qui sont consacrés à ses ennemis. Ce qui renvoie, d'abord, à la pauvreté des affects du paranoïaque, dont l'énergie est consacrée pour l'essentiel à observer l'autre et à interpréter son comportement; tandis que les interminables récits concernant les bizarreries, les conduites immorales, les troubles psychiques, les perversions sexuelles de tous les personnages de sa génération, et en particulier Jorge Ruan, Cayetano Corral et Carlos Bonaval, traduisent à la fois une forme d'obsession et un désir de jeter le discrédit sur ses prétendus persécuteurs. On peut aussi considérer comme significatives l'abondance et le contenu des digressions, parfois fort longues et fort pédantes. Ces considérations générales, fréquentes de toute manière dans le discours du paranoïaque, constituent en effet autant d'auto-justifications et/ou de dénégations (grossièrement) dissimulées sous la généralisation. Elles concernent le plus souvent les faiblesses de la mémoire (on ne saurait donc faire grief au narrateur, pas plus qu'à quiconque, des inévitables erreurs ou oublis que l'on repérerait dans son récit); le poids déterminant de l'enfance dans la personnalité adulte (le narrateur, qui a

${ }^{4}$ Voir la récurrence de termes tels que víctima, injusticia, exclusión, afrenta, reo, sospecha, recelo, indagar, prueba, demostrar, comprobación, venganza, etc.

${ }^{5}$ E. LEMBERT cité par Jacques Postel, Dictionnaire de psychiatrie, Paris, 1998, Larousse, p. 330a. 
souffert d'une l'infériorité liée à l'âge, est donc la victime du hasard qui l'a fait naître un peu trop tard); le caractère à la fois injuste et fallacieux de l'amour et de l'amitié (ce ne saurait donc être sa faute s'il a été dédaigné ou repoussé par Mary, Cayetano Corral, Jorge Ruan, Carlos Bonaval ; et par ailleurs la perte n'est donc pas bien grande). Ces digressions tendent en somme à dégager toute responsabilité personnelle du narrateur dans ses éventuels mensonges, dans son isolement affectif et dans son échec vital.

L'organisation du discours présente aussi des traits caractéristiques : la progression de la pensée correspond en effet exactement à celle de la « construction raisonnante ${ }^{6}$, et l'écriture proprement dite à celle de la paranoïa ${ }^{7}$. Quant à l'absence d'alinéa, elle ne prétend nullement reproduire le courant de conscience du narrateur, puisque son discours se situe tout à fait aux antipodes du monologue intérieur, non seulement par le contenu et la syntaxe, mais aussi par les marques de discours écrit et les références à un narrataire impersonnel qu'il semble important de convaincre $^{8}:$ il rappelle plutôt les libelles dénonciateurs par lesquels le paranoïaque tente volontiers de porter à la connaissance du public et/ou des autorités les plus diverses les persécutions dont il est l'objet. Il est en effet vital, selon la logique paranoïaque (du type «qui n'est pas avec moi est contre moi »), d'occuper le terrain de toutes les façons possibles, par la

6 «La complication conceptuelle progressive, la cascade d'explications et d'hypothèses auxiliaires surchargent toujours davantage l'enchevêtrement inextricable des arguties, des constats et des raisonnements. " Robert Pujol \& Henri Ey, Encyclopédie médicochirurgicale - Psychiatrie, Psychoses chroniques, III, "Les deux grands types de personnalités délirantes ", Paris, 1955, p. 6 b.

7 «[...] circonlocutions de la phrase : parenthèses, incidentes, subordinations intriquées, [...] reprises, redites, retours de la forme syntaxique. "Jacques Lacan, De la psychose paranoïaque dans ses rapports avec la personnalité (1932), Paris, 1996, Seuil, " Points », p. 179.

${ }^{8}$ Voir p. ex. : Se podrá pensar que ciertas expresiones corresponden menos a la realidad que al manifiesto disgusto con que siempre observé el culto [a Jorge Ruan] y la mitificación provocados por su memoria con posterioridad a su muerte. (p. 276) 
parole et/ou par l'écrit, afin de «recruter » un maximum d'alliés - forcément inconnus du narrateur en l'occurrence, puisque tous les personnages connus lui sont hostiles : d'où l'impersonnalité du narrataire.

Le point de vue narratif présente enfin une particularité qui va dans le même sens, car le narrateur se réfère parfois à des événements auxquels il n'a pas assisté, en pénétrant dans la conscience des protagonistes avec l'aisance d'un narrateur omniscient. Les cas les plus nets sont constitués par des scènes sexuelles narrées avec un grand luxe de détails et parfois des dialogues au discours direct, dont il n'a pu vraisemblablement entendre un récit aussi circonstancié ni même un récit quelconque. Et les indications du type " je suppose », " j'imagine », etc., disparaissent en général subrepticement pour laisser la place à un récit en quelque sorte très sûr de lui, où ces constructions de l'imagination du narrateur sont présentées en tout point comme des faits « réels », sans la moindre "critique » diraient les psychiatres. Or l'hypothèse paranoïaque permet d'interpréter ces infractions à la vision interne, plutôt que comme des dédoublements plus ou moins gratuits de la voix narrative, comme des manifestations du travail délirant : autrement dit, ce n'est pas là le discours d'un banal "menteur", mais bien plutôt de celui d'un " arrangeur " paranoïaque ${ }^{9}$, caractérisé par «la fausseté et l'invraisemblance flagrante du roman délirant $»^{10}$.

On observe d'ailleurs dans la fiction nombre d'éléments relevant du même type d'explication. Les motifs supposés de l'hostilité des trois « persécuteurs » principaux, en particulier, font plutôt penser à un délire d'interprétation : l'aversion qu'éprouve ou qu'éprouverait Carlos Bonaval à l'endroit du narrateur est attribuée à une vieille querelle parfaitement ridicule qui avait opposé leurs deux grands-pères (p. 183). Cayetano Corral, que ses goûts sexuels portent uniquement vers des «monstres », aurait pris ombrage des relations du narrateur avec la dénommée Rosa de Llanes (p. 227), qui n'entre pourtant pas dans cette catégorie de femmes (c'est

${ }^{9}$ La formule est du psychiatre F. LEURET (1834) cité par Pujol \& Ey, ibid., p. 4b-5a.

10 Sérieux \& Capgras cités par Lacan, ibid., p. 203. 
l'une des «contradictions » du récit). Cayetano Corral et Jorge Ruan, une fois devenus amis, l'auraient rejeté précisément parce qu'il avait voulu les réunir (p. 228). Et il faut ajouter à ces invraisemblances une « contradiction » flagrante et massive. Elle concerne la date de naissance du narrateur, dont on a dit l'importance dans l'interprétation qu'il propose de son échec personnel : en principe 1921 puisqu'il dit avoir " environ quinze ans » en 1936" (approximation curieuse mais qui passe sans doute inaperçue de la plupart des lecteurs, de même que l'inexactitude qui le fait se définir à cette époque non comme adolescent mais comme «enfant ${ }^{12}$ ). Mais l'un des souvenirs de sa petite enfance (un épisode très mineur de la vie familiale) se situe selon lui en $1920^{13}$, ce qui implique qu'il aurait eu plutôt vingt ans qu' "environ quinze » en 1936, et fait percevoir sa « passivité » d'alors non plus comme un comportement infantile mais comme la couardise d'un jeune adulte. La première date de naissance au contraire faisait apparaître l'accusation de pusillanimité comme l'une des manifestations d'injustice et de cruauté dont il serait la victime innocente, car comment « reprocher sa passivité » à un " enfant ", d'abord, puis à un être dont des circonstances adverses ont entravé la maturation, surtout lorsqu'on en a été comme Mary la cause au moins involontaire $^{14}$ ? Il s'agit donc ici, selon la même logique paranoïaque,

${ }^{11}$ Cuando a eso de los quince años me sentía yo a punto de entrar en aquel mundo que tanta impaciencia e inquietud me había procurado en los tres o cuatro anteriores... se derrumbó con la guerra civil. [...] Y quién sabe si por esa razón cuando trataba de constituirme en heredero de los desaparecidos, con pasos muy tímidos, y cuando por mis propios medios procuraba adentrarme en el terreno en que ellos me habrían llevado de la mano, de repente di media vuelta, renuncié al empeño, abandoné el lugar y no quise -hasta que ella volvió de América, sola- saber nada de lo que había dejado atrás. (p. 69)

12 Voir note 14.

${ }^{13}$ Todavía recuerdo - era yo muy niño - su llegada a la casa un día de verano a la caída de la tarde [...]. Ya entonces (yo creo que estoy hablando de 1920) [...]. (p. 172)

${ }^{14}$ Pero la misma que no había podido ni querido indagar la razón de las torpezas de aquel niño asustado, que la vio salir boquiabierto 
de «récuser le jugement d'autrui »15 afin de «prouver» qu'on est injustement persécuté.

Il faut encore ajouter à l'appui de l'hypothèse paranoïaque qu'elle permet de "découvrir », à partir des très rares données de l'histoire personnelle du narrateur, l'étiologie de ses troubles psychiques : le sentiment d'humiliation ${ }^{16}$ causé par sa position d'infériorité à l'intérieur de la famille nucléaire ${ }^{17}$, de la famille nucléaire à l'intérieur de la famille élargie ${ }^{18}$, et de la famille élargie par rapport aux autres familles bourgeoises de la contrée ${ }^{19}$ ) ; et même l' "étiologie sexuelle » déterminée par Freud, à savoir " la défense contre un désir homosexuel ${ }^{20}$, car outre que son choix d'objet (Mary) traduit une « incertitude du pragmatisme sexuel » ${ }^{21}$, il apparaît littéralement fasciné par ses prétendus rivaux Carlos Bonaval et Jorge Ruan, et ce dernier constitue un "doublet » de son frère Enrique, soit de l'être "de même sexe " auquel le narrateur «tient le plus profondément par son histoire affective ${ }^{22}$. Son hostilité aux deux hommes se ramènerait

hacia el exilio y boquiabierto la vio volver demudada y abatida ¿pretendería ahora inculparle de su pasividad? (p. 239)

15 Kraepelin cité par Lacan, ibid., p. 61.

16 «Les traits saillants [de la causation de la paranoïa] sont les humiliations, les rebuffades sociales, tout particulièrement chez l'homme. " (Sigmund Freud, Cinq psychanalyses, "Le président Schreber » (1911), Paris, 1967, PUF, "Bibliothèque de psychanalyse », p. 395)

${ }^{17}$ Le narrateur occupe dans sa fratrie une place intermédiaire reconnue comme défavorable, après au moins deux filles qui plus est (mis hermanas mayores, p. 16).

18 Son père est le moins considéré des gendres de la famille (p. 48).

${ }^{19}$ [...] su familia [del abuelo] rodeada por casi todas partes de una sociedad de rango más elevado [...]. (p. 8)

${ }^{20}$ FREUD, ibid., p. 305.

21 Par le «choix de partenaires d'une incompatibilité maxima", Lacan, ibid., p. 260.

22 «Le persécuteur principal est toujours de même sexe que le sujet, et est identique à, ou à tout le moins représente clairement, la personne du même sexe à laquelle le sujet tient le plus profondément par son histoire affective. » (Freud, ibid., p. 273) 
donc, selon la règle freudienne, à « contredire une proposition unique : 'Moi (un homme) je l'aime (lui, un homme)'» de toutes les manières possibles : "'Je ne l'aime pas - je le hais - parce qu'il me persécute' [...]. 'Ce n'est pas lui que j'aime - c'est elle que j'aime' [...]. 'Ce n'est pas moi qui aime l'homme - c'est elle qui l'aime' ».23

Toutefois, s'il est donc probable que tout le récit peut s'interpréter (ou se réinterpréter), tant globalement que dans le détail ${ }^{24}$, comme le "roman délirant »d'un paranoïaque, cette lecture «psychiatrique » n'exclut nullement a priori la lecture "policière » suggérée par Benet (elle l'encourage même plutôt lorsqu'on connaît la fréquence des conduites agressives chez les paranoïaques). Simplement il apparaît que l'examen le plus attentif échoue à découvrir des preuves de culpabilité du narrateur, même si les présomptions sont fortes face aux alibis éminemment suspects qu'il se donne comme négligemment à lui-même ${ }^{25}$. Autant dire que le lecteur qui se

\section{${ }^{23}$ FREUD, ibid., p. 308-309.}

24 On peut considérer comme symptomatique, p. ex., l'étrange "révélation" (après vingt ans!) concernant l'identité du premier amant de Mary, Carlos Bonaval ([...] así al reclinar mi cabeza sobre los zapatos para disimular mi detención acudió a ella la revelación de aquella identificación [...], p. 233-234), qui a toutes les apparences d'une "illusion de la mémoire " caractéristique de la paranoïa : i.e. d'une "insuffisance de la remémoration, qui permet à une imagefantasme (évoquée elle-même par les associations d'une perception, d'un rêve ou d'un complexe délirant) de se transformer en imagesouvenir" (Lacan, ibid., p 213). Le terme revelación (répété p. 238) est important car c'est l'un des "caractères propres à l'interprétation délirante " que de se présenter "comme une illumination spécifique " (Lacan, ibid., p. 211).

25 Lorsque le narrateur par exemple affirme avoir été absent lors de l'enterrement de Jorge Ruan (p. 64), on observe bien que le motif de l'un de ses "retours" aurait été le désir de revoir un ami "menacé d'une fin prochaine" qui ne peut être que Jorge Ruan (ibid.), mais celui-ci n'est pas forcément mort au cours de ce séjour. De même lorsqu'il suggère à plusieurs reprises que la destruction de la maison de Mary a été organisée par un certain Ruiz, ennemi juré de celle-ci et sans doute influencé par le père de Jorge Ruan, on remarque bien que Jorge Ruan qui servait d'intermédiaire aux deux hommes (p. 242, 343) est mort au moment des faits concernés, mais cela n'implique pas qu'ils aient perdu tout contact. Etc. 
risquerait sur la foi des propos de l'auteur à affirmer la culpabilité du personnage outrepasserait ses pouvoirs de détective amateur, puisque selon une pratique habituelle chez Benet le récit ne lui fournit pas toutes les pièces du puzzle fictionnel. L'examen du texte romanesque aura donc abouti à déceler chez le narrateur un délire paranoïaque auquel ne fait bien sûr allusion aucune déclaration paratextuelle de l'auteur, mais non à établir les faits fictifs objet du « coup de pouce auctorial à l'interprétation ${ }^{26}$. Or autant la lecture «psychiatrique » est fructueuse et divertissante (le lecteur ayant le sentiment d'avoir percé à jour un narrateur paranoïaque qui n'est pas parvenu à le « recruter »), autant la lecture "policière » est frustrante et irritante, puisque malgré son « intime conviction » le lecteur ne se sent pas autorisé à conclure à la culpabilité du narrateur comme il a pu conclure à sa paranoïa. Autrement dit, Benet s'est ingénié ici une fois de plus à égarer le lecteur, et il serait d'ailleurs intéressant de prolonger cette étude par celle des narrateurs impersonnels de ses autres romans, point si différents de l'«arrangeur » qui nous a occupés ici.

26 Gérard GENETTE, Seuils, Seuil, «Poétique », 1997, p. 368. 